\title{
Free Flap Protection with Halo Ring in the Occipital Scalp Region
}

\author{
Andreas Engel Krag, BSc (Med) ${ }^{1}$ Birgitte Jul Kiil, MD ${ }^{1}$ Jens Jakob Riis, MD \\ Gorm Burckhard von Oettingen, MD, PhD ${ }^{2}$ Gete Toft Eschen, MD ${ }^{1}$
}

\author{
${ }^{1}$ Department of Plastic Surgery, Aarhus University Hospital, Aarhus, \\ Denmark \\ ${ }^{2}$ Department of Neurosurgery, Aarhus University Hospital, Aarhus, \\ Denmark
}

J Reconstr Microsurg Open 2016;1:53-55.
Address for correspondence Andreas Engel Krag, BSc (Med), Department of Plastic Surgery, Aarhus University Hospital, Nørrebrogade 44, 8000 Aarhus C, Denmark (e-mail: andreas_engel_krag@hotmail.com).
Free flaps are applied to scalp and calvarial defects when local flap coverage is inadequate. Commonly used flaps are the free latissimus dorsi muscle flap and the free anterolateral thigh fasciocutaneous flap. ${ }^{1,2}$

Flap coverage of nonfrontal scalp regions is at risk of flap and pedicle compression, when patients are supine. This results in difficult patient positioning with risk of partial or complete flap necrosis. Hence, we have introduced perioperative halo ring placement on the reconstructed scalps, to avoid pressure on the flaps in the early postoperative weeks.

The PMT Model 1200 Series Halo (PMT Corporation, Chanhassen, MN), medium size, was applied reverted with the opening anteriorly. The ring is fixated on the skull with usually four titanium pins. Three consecutive cases are presented below with perioperative halo ring placement in microsurgical scalp reconstruction.

\section{Case 1}

A 68-year-old man was admitted with nonmalignant chronic scalp ulcerations and cranial bone necrosis in the left parietal and occipital region. In childhood, the patient had been exposed to an unintended overdose of radiotherapy for tinea capitis. Affected scalp and skull were excised leaving a $10 \times 14 \mathrm{~cm}$ defect with dura preserved. The defect was covered with a free latissimus dorsi muscle flap anastomosed to the right facial artery and vein with vein grafting. Split skin grafts were placed on the muscle flap. Postoperatively, a distal part of the flap in the occipital region was lost due to pressure necrosis, from when the patient had been lying down or sitting, resulting in cranial bone exposure (-Fig. 1).

Thirty-five days later, the exposed bone was successfully covered with a free rectus abdominis flap anastomosed to the left facial artery and vein with two vein grafts, and split skin grafts were placed on the muscle. A halo ring was placed perioperatively to avoid pressure on the flap and pedicle. After 17 days, the halo ring was removed and both muscle flaps healed without further complications.

\section{Case 2}

A 45-year-old man was referred with chronic open wounds and dural defects in the occipital region, following several resections of radiation-induced meningioma (-Fig. 2). After a failed procedure with a rotation flap, the defect was successfully covered with a free latissimus dorsi muscle flap and split skin grafts. The thoracodorsal vessels were anastomosed to the right superficial temporal artery and vein, and in the end of the procedure a halo ring was applied. Twenty-seven days postsurgery, the halo ring was removed. Six weeks postsurgery, the flap had healed without complications (-Fig. 3 ).

\section{Case 3}

A 67-year-old woman was admitted with recurrent malignant schwannoma in the right parietal scalp region. The patient had previously undergone nonradical tumor resection and reconstruction with a cervical rotation flap, a free radial forearm flap, and a free latissimus dorsi flap at another center. Scalp tissue, bone, and dura were excised in a region posterior to the right ear from the superior sagittal sinus to the transverse sinus. The dural defect was reconstructed with acellular dermal matrix. A free latissimus dorsi muscle flap was harvested and anastomosed to the left occipital artery and left external jugular vein, and a halo ring was applied at the end of the procedure. In this high-risk patient, there was a considerable risk of reoperation and hence the flap was not covered with split skin received

August 8, 2015

accepted after revision

September 27, 2015

published online

January 18, 2016
DOI http://dx.doi.org/

10.1055/s-0035-1570508. ISSN 2377-0813.
Copyright $\odot 2016$ by Thieme Medical Publishers, Inc., 333 Seventh Avenue, New York, NY 10001, USA. Tel: +1(212) 584-4662.
License terms

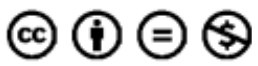




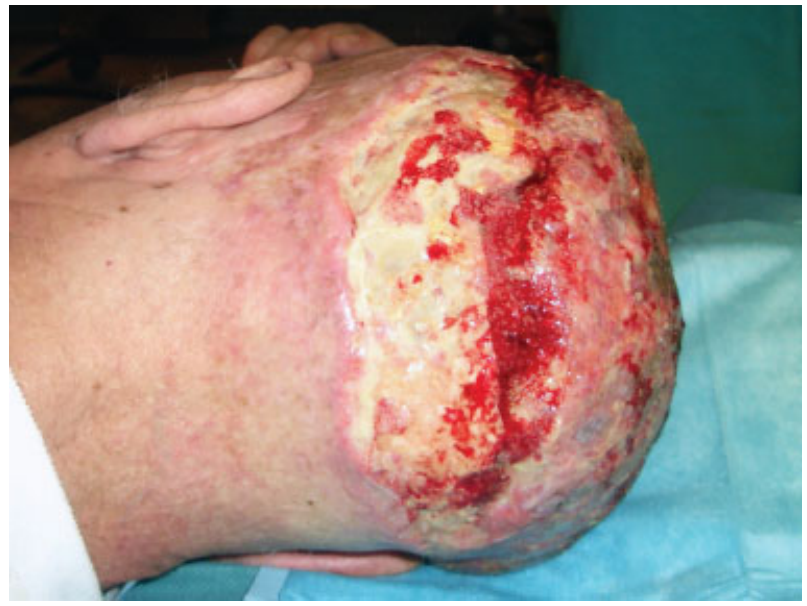

Fig. 1 Case 1: Distal necrosis of the latissimus dorsi muscle flap resulted in cranial bone exposure occipitally.

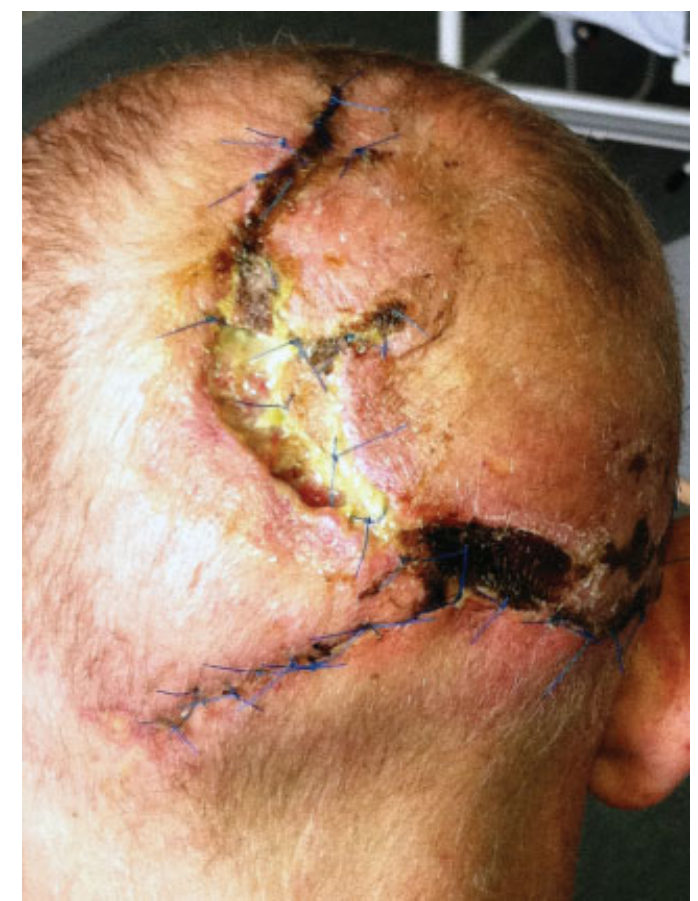

Fig. 2 Case 2: The patient was referred with chronic open wounds including dural defects in the occipital scalp region.

grafts until the following day in the ward. Postoperatively the patient went into respiratory failure and delirium and was sedated for 4 days in the intensive care unit but recovered completely. Twenty-seven days after surgery, the flap had healed and the halo ring was removed ( - Fig.4).

\section{Discussion}

The halo fixation device is typically applied for posttraumatic or postoperative immobilization of the cervical spine. ${ }^{3}$ Few case reports have described application of the halo for protection of scalp reconstructions. ${ }^{4-6}$

By applying the halo ring to patients undergoing free flap scalp reconstruction, we have been able to minimize pressure on the flaps and vascular pedicles and provide optimal healing

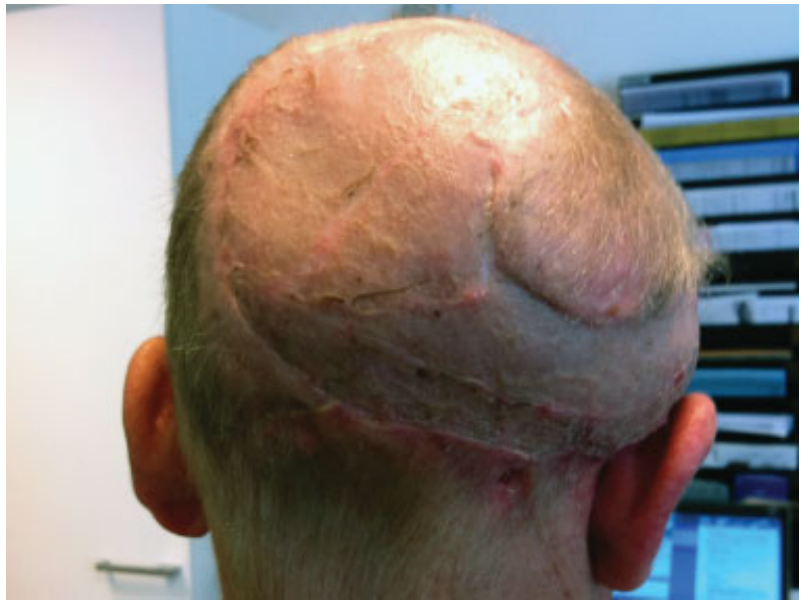

Fig. 3 Case 2: 6 weeks after the operation the flap had healed without complications.

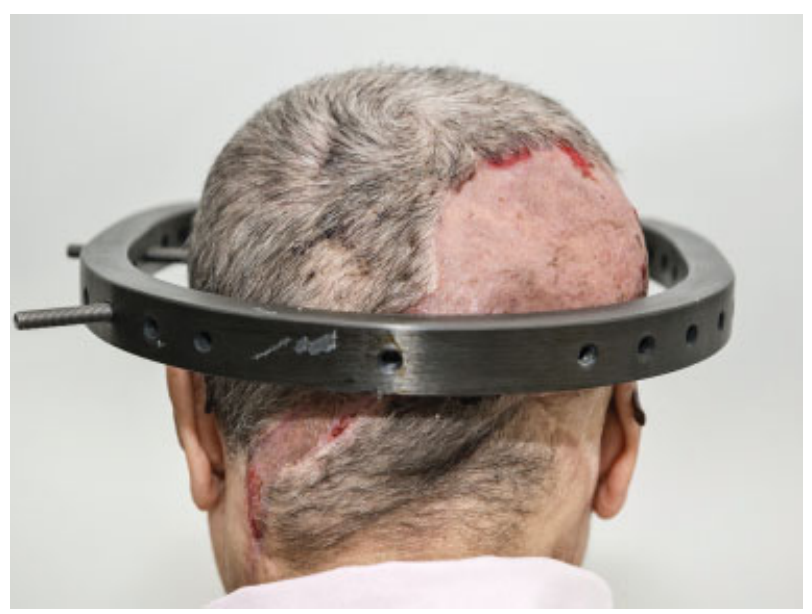

Fig. 4 Case 3: The halo ring and healed flap (27 days postoperatively).

conditions. Especially in patients who have undergone neurosurgical intervention, we find the halo ring applicable, since the patient might be sedated and immobilized in the immediate postoperative period and critical flap healing phase. The space between the halo ring and flap makes room for wound care and delayed placement of split skin grafts on muscle flaps, as well as optimal conditions for clinical flap observation.

Risks are related to placement of the titanium pins fixating the halo ring. The most common complications are aseptic pin loosening and pin tract infection, which occur in 13 to $36 \%$ and 5 to $20 \%$ of cases, respectively. ${ }^{7,8}$ Less common complications are compression or damage to the supraorbital or supratrochlear nerves; and rare cases with pin penetration through the skull and formation of cerebral abscesses have been reported. ${ }^{7,9}$ To avoid pin site infection, our patients regularly had their pinholes checked by nurses, and no complications occurred related to halo ring placement.

Placing the anterior pins in the safe zone $1 \mathrm{~cm}$ above the most lateral eyebrow reduces the risk of supraorbital or supratrochlear nerve damage. To avoid cranial penetration, the anterior pins should be placed anteriorly to the temporal muscle and fossa with thin cranial bone. There are not the same neuromuscular restrictions with regard to placement of 
the posterior pins, and skull thickness does not vary greatly posteriorly. ${ }^{7,10}$ The pins should be placed at, or just below, the skull equator to obtain a perpendicular angle between the pin and skull to minimize pin loosening. ${ }^{7}$ Furthermore, pin placement can be adjusted with respect to cranial bone defects for the correct placement of four to six pins, as the halo ring has 20 pinholes. We always evaluate the patients' computed tomography scans of cerebrum for judgment of skull thickness and optimal placement of pins.

The halo ring used for protection of microsurgical scalp reconstructions diminishes the risk of flap necrosis. We recommend the halo ring to be applied in the operating room immediately after microsurgical reconstruction.

\section{Conflict of Interest}

None.

Funding

None.

\section{Acknowledgments}

The authors thank Tonny Foghmar, photographer at Aarhus University Hospital, Denmark, for providing photographs.

\section{References}

1 Chao AH, Yu P, Skoracki RJ, Demonte F, Hanasono MM. Microsurgical reconstruction of composite scalp and calvarial defects in patients with cancer: a 10-year experience. Head Neck 2012; 34(12):1759-1764

2 Fischer JP, Sieber B, Nelson JA, et al. A 15-year experience of complex scalp reconstruction using free tissue transferanalysis of risk factors for complications. J Reconstr Microsurg 2013;29(2):89-97

3 Nickel VL, Perry J, Garrett A, Heppenstall M. The halo. A spinal skeletal traction fixation device. J Bone Joint Surg Am 1968;50(7): 1400-1409

4 Sandow MJ, Hamilton RB, Heden PG. A modified halo frame to assist omentum transfer to the scalp. Br J Plast Surg 1985;38(2): 288-291

5 Schubert W, Guzman-Stein G, Hope R, Ahrenholz DH, Solem LD, Cunningham BL. Scalping injuries: new technique for stabilization of flaps to the skull. Plast Reconstr Surg 1988;81(5):780-782

6 Fritsche E, Helaly P. Successful scalp replantation-a case report [in German]. Handchir Mikrochir Plast Chir 1995;27(6):319-321

7 Garfin SR, Botte MJ, Waters RL, Nickel VL. Complications in the use of the halo fixation device. J Bone Joint Surg Am 1986;68(3): 320-325

8 Bransford RJ, Stevens DW, Uyeji S, Bellabarba C, Chapman JR. Halo vest treatment of cervical spine injuries: a success and survivorship analysis. Spine 2009;34(15):1561-1566

9 Saeed MU, Dacuycuy MA, Kennedy DJ. Halo pin insertion-associated brain abscess: case report and review of literature. Spine 2007;32(8):E271-E274

10 Garfin SR, Botte MJ, Centeno RS, Nickel VL. Osteology of the skull as it affects halo pin placement. Spine 1985;10(8):696-698 